\title{
Surgical management and long-term outcomes for acute ascending aortic dissection
}

\author{
Louis-Mathieu Stevens, MD, ${ }^{\mathrm{a}}$ Joren C. Madsen, MD, DPhil, ${ }^{\mathrm{a}}$ Eric M. Isselbacher, MD, ${ }^{\mathrm{b}}$ Paul Khairy, MD, \\ $\mathrm{PhD},{ }^{\mathrm{c}}$ Thomas E. MacGillivray, MD, ${ }^{\mathrm{a}}$ Alan D. Hilgenberg, MD, ${ }^{\mathrm{a}}$ and Arvind K. Agnihotri, $\mathrm{MD}^{\mathrm{a}}$
}

Objective: We sought to assess early and late survival and cardiovascular-specific mortality after surgical repair of acute ascending aortic dissection and the effect of differences in surgical technique, patient characteristics, and preoperative diagnostic testing.

Methods: Between 1979 and 2003, 195 consecutive patients underwent repair for acute ascending aortic dissection within 2 weeks of the onset of symptoms. Mean follow-up was $7.0 \pm 5.9$ years (range, 0-26 years) and was $100 \%$ complete.

Results: Patients were aged $62 \pm 15$ years on average and were mostly male (66\%) and hypertensive (69\%). Risk of death early and late after the operation decreased over the study period, with hospital mortality decreasing from $21 \%$ to $4 \%$ when comparing the first and most recent quartiles $\left(P=.007, \chi^{2}\right.$ test for trend). At $1,5,10$, and 20 years postoperatively, survival was $84 \%, 69 \%, 55 \%$, and $30 \%$, respectively, and freedom from cardiovascular death was $86 \%, 80 \%, 71 \%$, and $51 \%$, respectively. Additional independent risk factors for death were older age $(P<.001)$, renal dysfunction $(P<.003)$, syncope $(P=.007)$, and peripheral vascular disease $(P=.006)$. During the study period, echocardiographic and computed tomographic diagnostic imaging replaced routine aortic angiographic analysis, and operative techniques involved more frequent use of open distal anastomoses, retrograde cerebral perfusion, earlier restoration of antegrade perfusion, and a conservative approach to aortic arch repair. Freedom from reoperation on the aorta or aortic valve was $93 \%$ and $84 \%$ at 5 and 10 years, respectively.

Conclusions: Early and late survival after repair of acute ascending aortic dissection has improved progressively over 25 years in association with noticeable changes in preoperative and intraoperative management. Aortic reoperations were infrequent during follow-up. (J Thorac Cardiovasc Surg 2009;138:1349-57)

Supplemental material is available online.

Patients presenting with ascending aortic dissection within 2 weeks of the onset of symptoms are considered to have acute ascending aortic dissection (AAAD), whereas chronic presentation is defined when there is at least 2 weeks from the onset of symptoms. The importance of this diagnostic time frame is underscored by the reported $65 \%$ to $75 \%$ death rate of patients associated with untreated AAAD. ${ }^{1}$ Hospital mortality for repair of AAAD has decreased since the $40 \%$ mortality rate reported by Debakey and colleagues in $1965^{2}$

\footnotetext{
From the Divisions of Cardiac Surgery ${ }^{\mathrm{a}}$ and Cardiology, ${ }^{\mathrm{b}}$ Massachusetts General Hospital, Boston, Mass; and the Department of Medicine, ${ }^{\mathrm{c}}$ Montreal Heart Institute, Montreal, Quebec, Canada.

Disclosures: Salary support (to L.M.S.) was provided by a fellowship award from the Canadian Institutes of Health Research's Clinical Research Initiative and the Rosetti Fund (Massachusetts General Hospital, Boston, Massachusetts).

Received for publication May 2, 2008; revisions received Nov 6, 2008; accepted for publication Jan 4, 2009; available ahead of print June 1, 2009.

Address for reprints: Arvind K. Agnihotri, MD, Department of Cardiothoracic Surgery, Massachusetts General Hospital, 55 Fruit St, COX 630, Boston, MA 02114 (E-mail: aagnihotri@partners.org).

$0022-5223 / \$ 36.00$

Copyright (C) 2009 by The American Association for Thoracic Surgery

doi:10.1016/j.jtcvs.2009.01.030
}

and now ranges from 5\% to $26 \%{ }^{1,3-18}$ Among studies reporting long-term results after repair of AAAD, few reports are based on a mean follow-up of greater than 5 years, with 10 -year survival estimates ranging from $37 \%$ to $71 \%$. $^{4,7,8,19,20}$

This study sought to extend information regarding longterm cardiovascular and all-cause survival after repair of AAAD by means of review of the experience in a single institution over a 26-year period. Additionally, we sought to assess the effect of patient characteristics and explore the changes in outcome over time that might have occurred with changes in preoperative evaluation and surgical techniques.

\section{MATERIALS AND METHODS \\ Patients}

A retrospective study was undertaken of patients undergoing surgical repair of AAAD from October 1979 to December 2003. A database of all cardiac surgical operations performed at Massachusetts General Hospital was queried for mention of aortic dissection. Two hundred seventy-three patients were reviewed. We excluded $76(28 \%)$ patients for the following reasons: chronic dissection $(\mathrm{n}=35[13 \%])$, descending aortic dissection (DeBakey type III/Stanford type B, $\mathrm{n}=34$ [12\%]), and intraoperative aortic dissection or traumatic aortic injuries $(n=7[3 \%])$. Two patients, excluded from this analysis, were considered inoperable after surgical exploration because of extensive dissection in the descending aorta with multiple rupture sites, cardiac arrest before cardiopulmonary bypass, or both. The 


\section{Abbreviations and Acronyms \\ $\mathrm{AAAD}=$ acute ascending aortic dissection \\ $\mathrm{AAR}=$ ascending aortic replacement \\ $\mathrm{CT}=$ computed tomography}

final cohort consisted of 195 patients who underwent surgical intervention for ascending aortic dissection within 2 weeks of symptom onset.

Patient demographics, comorbidities, operative details and postoperative outcomes were extracted retrospectively by means of chart review (see Appendix E1). Involvement of the aorta and its branches in the dissection process was determined by means of preoperative imaging and intraoperative assessment of the dissection process. Ascending aortic repair was completed with conservative techniques adapted to the dissection extension and involvement of the aortic valve, including ascending aortic replacement (AAR) without aortic valve replacement if aortic valve reconstruction was feasible or the disease process was limited to the ascending aorta above the sinotubular junction, AAR with aortic valve replacement if the aortic valve was dysfunctional and the aortic sinuses were not involved, and AAR with composite root replacement otherwise. ${ }^{21}$ In general, aortic arch repair was performed only when the intimal tear extended in the aortic arch. Hemiarch repair was defined as a replacement that included the lesser curvature of the aortic arch beyond the level of the innominate artery but did not involve the aortic arch vessels. Total arch repair involved replacement of the anterior and posterior aortic arch, with reimplantation of the branch vessels either as an island or as individual branch grafts. Antegrade perfusion refers to restoration of antegrade cerebral and corporal perfusion through the aortic graft, aortic arch, or subclavian artery after distal aortic graft anastomosis.

Survival follow-up data were obtained by linking to the National Death Index for all patients up to December 31, 2005. Date and cause of death were confirmed with National Death Index data. Aortic reoperation follow-up was obtained by means of telephone interview, mailed questionnaires to patients, queries to family and primary care physicians/cardiologists, queries to patient records, and correspondence. Aortic reoperation was defined as any surgical procedure performed on the thoracic or abdominal aorta or aortic valve after patient discharge from index admission. Ethics approval for this study was obtained from the institutional review board.

Hospital mortality included death during the hospitalization and death within 30 days of the operation. Cardiovascular death included hospital death for index admission and follow-up death caused by cardiac ischemic disease, endocarditis, other cardiac valve diseases, or vascular disease, including stroke and vascular surgery-related deaths. Hypertension was recorded if the patient was given a clinical diagnosis of hypertension or was treated with antihypertensive medication before the index admission. Peripheral vascular disease comprised any known atheromatous involvement of systemic arteries with the exception of the coronary arteries and included cerebrovascular disease, abdominal aortic atheromatosis and/or aneurysmal disease, intermittent claudication, or previous vascular surgery. Marfan syndrome was defined according to the 1996 revised criteria, ${ }^{22}$ and histopathologic confirmation was sought when possible. Most patients were hypertensive after dissection. Cardiogenic shock was reported if the preoperative systolic blood pressure was less than $80 \mathrm{~mm} \mathrm{Hg}$, the cardiac index was less than $1.8 \mathrm{~mL} / \mathrm{m}^{2}$, or they required intravenous use of inotropes. Patients were considered unstable if they experienced accelerating pain or hemodynamic instability (uncontrollable hypertension or hypotension). Salvage was applied if patients were undergoing cardiopulmonary resuscitation en route to the operating room or before anesthesia induction.

\section{Statistical Analysis}

All statistical analyses were performed with SAS release 9.1 software (SAS Institute, Inc, Cary, NC). Data are expressed as means \pm standard deviations or medians and ranges for continuous variables and as numbers (percentages) for categorical variables. Univariate analyses included the 2-tailed Wilcoxon rank sum or $t$ tests for continuous variables and the Fisher's exact test for discrete variables (see Appendix E1 for the list of variables). For continuous variables, the linearity assumptions were assessed for all models and transformations considered, where appropriate. For multivariate analyses, missing data were handled by means of dummy coding for dichotomous covariates. Covariates with more than $5 \%$ missing data were discarded from the multivariate analyses. There were no missing data for continuous covariates. The stability of effect estimates in the final model was assessed by completing a bootstrap procedure with 5000 random samples of 195 patients chosen with replacement from the original dataset. The standard deviations of the means of the bootstrap estimates were used to test the original parameter estimates from the final model by means of a $\chi^{2}$ test.

Several variables were strongly associated with the year of surgical intervention (and with each other) and were, by necessity, excluded from the multivariate analysis (see the Results section). These were related to preoperative or treatment variables that became available during the study period and thus might not have been possible or were very difficult to apply in the early part of the series. To assess competing diagnostic or surgical strategies across time, we defined mutually exclusive categories, such as angiographic analysis alone, angiographic analysis with computed tomographic (CT) scanning, angiographic analysis with echocardiographic analysis, CT scanning alone, and so on. As part of this exploratory analysis, a multinomial logit model was fitted by using the categories as the outcome variable and the date of surgical intervention as the independent variable, thereby producing predicted probabilities across time, each representing the likelihood of being assigned to one of the categories (and summing to $100 \%$ at each time point). Categories with a similar trend, few cases, or both were combined if clinically meaningful, and the model was refitted.

Survival and aortic reoperation were estimated by using the Kaplan-Meier technique, with log-rank tests to compare stratified groups. Time-specific effects were estimated with parametric, multiphase (mixture distribution components) hazard regression by using analytic tools available from the Cleveland Clinic Heart Center Hazard Web site (http://www.cleveland clinic.org/heartcenter/hazard/default.htm). A nonautomated directed technique of stepwise variable entry was used. Multiphase parametric hazard regression was used, allowing assessment of the effect of baseline predictors on different phases of follow-up separately. Kaplan-Meier and parametric estimates of the survival of patients discharged alive (Weibull distribution) were compared with the survival of a cohort from Massachusetts matched for age, sex, and race by using 1989-1991 state lifetables. ${ }^{23}$ Risk factors for aortic reoperation after discharge were assessed by using a Cox model.

\section{RESULTS \\ Presentation}

Patient characteristics, operative data, aortic repair techniques, and concomitant procedures are reported in Table 1. Twenty-seven $(14 \%)$ patients had prior aortic disease; $25(13 \%)$ patients had prior aortic valve dysfunction, a bicuspid aortic valve, or both; and 31 patients had prior cardiovascular surgery (7 underwent coronary artery bypass grafting, 10 underwent valve surgery, 10 underwent major vascular surgery, and 4 underwent other procedures). Thirteen $(7 \%)$ patients were given diagnoses of Marfan syndrome. Electrocardiographic findings were most often nonspecific (44\%), but $42(23 \%)$ patients presented with either ST-segment elevation or depression. Nineteen $(10 \%)$ patients underwent coronary artery catheterization before surgical intervention. 
TABLE 1. Patient characteristics, operative data, and hospital mortality

\begin{tabular}{|c|c|c|c|c|c|}
\hline & \multirow[b]{2}{*}{ Overall } & \multicolumn{4}{|c|}{ Hospital mortality* } \\
\hline & & Exposed & Unexposed & Odds ratio $(95 \% \mathrm{CI})$ & $P$ value \\
\hline Overall & $195(100 \%)$ & $26 / 195(13 \%)$ & - & - & - \\
\hline \multicolumn{6}{|l|}{ Patient characteristics } \\
\hline Age (y) & $62 \pm 15$ & - & - & $1.84 \dagger(1.26-2.70)$ & .002 \\
\hline Male sex & $128(66 \%)$ & $16 / 128(13 \%)$ & $10 / 67(15 \%)$ & $0.81(0.35-1.91)$ & 6 \\
\hline Hypertension & $134(69 \%)$ & $21 / 134(16 \%)$ & $5 / 61(8 \%)$ & $2.08(0.75-5.81)$ & .2 \\
\hline Coronary artery disease & $32(16 \%)$ & $6 / 32(19 \%)$ & $20 / 163(12 \%)$ & $1.65(0.60-4.50)$ & .3 \\
\hline Chronic lung disease & $23(12 \%)$ & $6 / 23(26 \%)$ & $20 / 172(12 \%)$ & $2.68(0.95-7.60)$ & .06 \\
\hline Peripheral vascular disease & $38(19 \%)$ & $5 / 38(13 \%)$ & $21 / 157(13 \%)$ & $0.98(0.34-2.80)$ & $>.9$ \\
\hline $\begin{array}{l}\text { Serum creatinine }(\mathrm{mg} / \mathrm{dL}) \text { level } \\
\text { at admission }\end{array}$ & $1.24 \pm 0.51$ & - & - & $2.47(1.26-4.84)$ & .009 \\
\hline Prior cardiac valve dysfunction & $35(18 \%)$ & $3 / 35(9 \%)$ & $23 / 160(14 \%)$ & $0.56(0.16-1.97)$ & .4 \\
\hline \multicolumn{6}{|l|}{ Presenting symptoms } \\
\hline Sudden-onset pain & $144 / 190(76 \%)$ & $18 / 144(13 \%)$ & $8 / 46(17 \%)$ & $0.68(0.27-1.68)$ & .4 \\
\hline Anterior chest pain & $143 / 190(75 \%)$ & $17 / 143(12 \%)$ & $9 / 47(19 \%)$ & $0.57(0.23-1.38)$ & .2 \\
\hline Head/neck pain & $45 / 190(24 \%)$ & $3 / 45(7 \%)$ & $23 / 145(16 \%)$ & $0.38(0.11-1.33)$ & .1 \\
\hline Back pain & $93 / 190(49 \%)$ & $10 / 93(11 \%)$ & $16 / 97(16 \%)$ & $0.61(0.26-1.42)$ & .3 \\
\hline Abdominal pain & $39 / 190(21 \%)$ & $3 / 39(8 \%)$ & $23 / 151(15 \%)$ & $0.46(0.13-1.63)$ & .2 \\
\hline \multicolumn{6}{|l|}{ Clinical status } \\
\hline Hemodynamic status & & & & & .02 \\
\hline Stable (reference) & $112(57 \%)$ & $9 / 112(8 \%)$ & - & 1 & \\
\hline Unstable & $71(36 \%)$ & $13 / 71(18 \%)$ & - & $2.57(1.03-6.37)$ & \\
\hline Salvage & $12(6 \%)$ & $4 / 12(33 \%)$ & - & $5.72(1.44-22.75)$ & \\
\hline Cardiogenic shock & $45(23 \%)$ & $11 / 45(24 \%)$ & $15 / 150(10 \%)$ & $2.91(1.23-6.91)$ & .02 \\
\hline Tamponade & $26(13 \%)$ & $6 / 26(23 \%)$ & $20 / 169(12 \%)$ & $2.24(0.80-6.23)$ & .1 \\
\hline Syncope & $33(17 \%)$ & $12 / 33(36 \%)$ & $14 / 162(9 \%)$ & $6.04(2.47-14.80)$ & $<.001$ \\
\hline Altered mental status & $23(12 \%)$ & $5 / 23(22 \%)$ & $21 / 172(12 \%)$ & $2.00(0.67-5.95)$ & .2 \\
\hline $\begin{array}{l}\text { Focal neurologic } \\
\text { deficit preoperatively }\end{array}$ & $12(6 \%)$ & $3 / 12(25 \%)$ & $23 / 183(13 \%)$ & $2.32(0.58-9.20)$ & .2 \\
\hline $\begin{array}{l}\text { ECG ST—-segment elevation } \\
\text { or ST—-segment depression }\end{array}$ & $42 / 186(23 \%)$ & $8 / 42(19 \%)$ & $17 / 144(12 \%)$ & $1.76(0.70-4.42)$ & .2 \\
\hline \multicolumn{6}{|l|}{ Arteries involved in the dissection process } \\
\hline DeBakey type I & $162(83 \%)$ & $20 / 162(12 \%)$ & $6 / 33(18 \%)$ & $0.63(0.23-1.72)$ & .4 \\
\hline DeBakey type II & $33(17 \%)$ & $6 / 33(18 \%)$ & $20 / 162(12 \%)$ & $1.58(0.58-4.29)$ & \\
\hline Coronary arteries & $40(21 \%)$ & $6 / 40(15 \%)$ & $20 / 155(13 \%)$ & $1.19(0.44-3.20)$ & .7 \\
\hline Carotid arteries & $43(22 \%)$ & $8 / 43(19 \%)$ & $18 / 152(12 \%)$ & $1.70(0.68-4.24)$ & .3 \\
\hline \multicolumn{6}{|l|}{ Surgical data } \\
\hline Interval symptom-operation (h) & $24(0.9-480)$ & - & - & $1.01(0.90-1.14)$ & .9 \\
\hline CPB time (min) & $166 \pm 70$ & - & - & $1.02 \ddagger(0.96-1.07)$ & .6 \\
\hline Crossclamp time (min) & $108 \pm 44$ & - & - & $0.93 \ddagger(0.83-1.05)$ & .2 \\
\hline Circulatory arrest time (min) & $32 \pm 15$ & - & - & $0.81 \ddagger(0.54-1.23)$ & .3 \\
\hline Open distal anastomosis & $146(75 \%)$ & $15 / 146(10 \%)$ & $11 / 49(22 \%)$ & $0.40(0.17-0.93)$ & .03 \\
\hline Retrograde cerebral perfusion & $24(12 \%)$ & $0 / 24(0 \%)$ & $26 / 171(15 \%)$ & - & - \\
\hline $\begin{array}{l}\text { Antegrade cerebral perfusion } \\
\text { through arch or graft }\end{array}$ & $56(29 \%)$ & $5 / 56(9 \%)$ & $21 / 139(15 \%)$ & $0.55(0.20-1.54)$ & .3 \\
\hline Concomitant hemiarch repair & $18(9 \%)$ & $2 / 18(11 \%)$ & $24 / 177(14 \%)$ & $0.80(0.17-3.69)$ & .8 \\
\hline Concomitant total arch repair & $5(3 \%)$ & $1 / 5(20 \%)$ & $25 / 190(13 \%)$ & $1.65(0.18-15.36)$ & .7 \\
\hline Concomitant CABG & $14(7 \%)$ & $3 / 14(21 \%)$ & $23 / 181(13 \%)$ & $1.87(0.49-7.22)$ & .4 \\
\hline $\begin{array}{l}\text { Antifibrinolytic use (aprotinin } \\
\text { or aminocaproic acid [Amicar]) }\end{array}$ & $108(55 \%)$ & $7 / 108(6 \%)$ & $19 / 87(22 \%)$ & $0.25(0.10-0.62)$ & .003 \\
\hline Surgical glue use & $44(23 \%)$ & $1 / 44(2 \%)$ & $25 / 151(17 \%)$ & $0.12(0.02-0.89)$ & .04 \\
\hline Year of operation (since October 1, 1979) & $15 \pm 7$ & - & - & $0.65 \S(0.48-0.88)$ & .005 \\
\hline
\end{tabular}

$C I$, Confidence interval; $E C G$, electrocardiography; $C P B$, cardiopulmonary bypass; $C A B G$, coronary artery bypass grafting. *Hospital mortality in patients exposed (presence of) or unexposed (absence of) to each categorical risk factor (eg, male vs female sex or hypertensive vs nonhypertensive) with corresponding odds ratios and univariable $P$ values from logistic regression models. †Effect for 10-year difference in age. †Effect for 10-minute difference in cardiopulmonary bypass, crossclamp, or circulatory arrest times. $\S$ Effect for 5 year difference in year of operation. 


\section{Surgical Techniques}

Ascending aortic and aortic valve surgical proceduresincluded $137(60 \%)$ ascending aortic grafts, including aortic valve resuspension; 47 (24\%) composite valve and ascending aortic grafts (39 mechanical and 8 biologic valves); 9 $(5 \%)$ ascending aortic grafts and aortic valve replacements (6 mechanical and 3 biologic valves); and 2 primary repairs (aneurysmoplasty without aortic graft). The average ascending aortic graft was $28 \pm 3 \mathrm{~mm}$, and the median aortic valve replacement size was $27 \mathrm{~mm}$ (range, 19-31 mm).

Initial arterial cannulation was most commonly performed at the femoral artery $(96 \%)$ and rarely at the axillary artery $(2 \%)$ or aortic arch $(2 \%)$. Venous cannulation was performed by using a 2 -staged right atrial canula $(64 \%)$ or bicaval cannulas $(26 \%)$, through the femoral vein $(31 \%)$, or both. Most patients $(98 \%)$ were given cold intermittent blood cardioplegia $(98 \%)$, and $3(2 \%)$ patients received warm continuous blood cardioplegia. Hypothermic circulatory arrest was used in $148(76 \%)$ patients, and the circulatory arrest lasted $32 \pm 15$ minutes, with a minimal core temperature of $17^{\circ} \mathrm{C} \pm 4^{\circ} \mathrm{C}$.

\section{Early Results}

Hospital mortality over the entire study period was $13 \%$ (26 patients) but improved dramatically during the study interval. When the study period was divided into 4 equal subgroups of consecutive patients, we observed a gradual decrease in hospital mortality: $21 \%(10 / 48,1979-1990)$, $18 \%(9 / 49,1990-1996), 10 \%(5 / 49,1996-2000)$, and $4 \%\left(2 / 49,2000-2003 ; P=.007, \chi^{2}\right.$ test for trend). Intraoperative deaths occurred in 6 patients (23\% of deaths) and were due to uncontrollable hemorrhage $(\mathrm{n}=3)$, post-cardiopulmonary bypass heart failure $(\mathrm{n}=2)$, or pulmonary artery rupture $(\mathrm{m}=1)$. Postoperative deaths were caused by heart failure $(n=3)$, massive hemorrhage $(n=2)$, stroke $(n=9)$, renal failure $(\mathrm{n}=1)$, and multiple system failure $(\mathrm{n}=5)$. Univariate predictors for hospital mortality are presented in Table 1. Independent predictors of hospital mortality are reported in Table 2.*

In-hospital complications included cardiac reoperation in $35(18 \%)$ patients, delirium in $50(26 \%)$ patients, persistent neurologic dysfunction in $37(19 \%)$ patients, pneumonia documented by positive sputum culture and chest imaging in $42(22 \%)$ patients, pleural effusion in $37(19 \%)$ patients, and temporary or permanent dialysis in $18(9 \%)$ patients. Most patients received transfusion of packed red blood cells (use, $92 \%$; median, 6 units), platelets (use, $82 \%$; median, 15

\footnotetext{
* Marfan syndrome was initially selected as a significant independent predictor of hospital mortality (odds ratio, 24.61; 95\% confidence interval, $2.08-291.1 ; P=.01$ ), but the variable was ultimately dropped from the list of independent risk factors after we found that the stability of this estimate was in question. With the bootstrap procedure, the variable was rejected with a $P$ value of .25 ; all other estimated $P$ values remained significant at a $P$ value of less than .001 . The final model had a c-statistics of 0.85 and a Hosmer-Lemeshow goodness-of-fit test $P$ value of .6.
}

units), and fresh frozen plasma or cryoprecipitate (use, $86 \%$; median, 8 units). Patients with stroke had a longer postoperative length of stay $(P=.02)$, and their hospital mortality was 2.3 times higher compared with that seen in patients without stroke ( $24 \%$ vs $11 \%, P=.03)$. Independent predictors of postoperative stroke are listed in Table 3 . The mean maximum postoperative creatinine level was $2.3 \pm 2.0$ $\mathrm{mg} / \mathrm{dL}$ and decreased to $1.3 \pm 1.0 \mathrm{mg} / \mathrm{dL}$ on discharge or death. Hospital mortality was 2.9 times higher among patients requiring dialysis postoperatively compared with those who did not undergo dialysis $(33 \%$ vs $11 \%, P=$ $.02)$. Of the 169 patients discharged alive, $93(55 \%)$ were discharged home, including 24 who were visited by a nurse, with the remaining $76(45 \%)$ patients discharged to a rehabilitation center.

\section{Long-Term Survival}

The mean follow-up was $7.0 \pm 5.9$ years (range, 1 day-26 years), or $8.1 \pm 5.6$ years in patients discharged alive, and was $100 \%$ complete. Overall survival was $84 \%, 69 \%$, $55 \%, 48 \%$, and $30 \%$ at $1,5,10,15$, and 20 years, respectively, and freedom from cardiovascular death was $86 \%$, $80 \%, 71 \%, 67 \%$, and $51 \%$ at $1,5,10,15$, and 20 years, respectively (Figure 1). Figure 1 also shows the high early risk of death followed by a low but slowly increasing late hazard phase. Late deaths were due to ischemic cardiac diseases ( $\mathrm{n}$ $=14[22 \%])$; cerebrovascular disease $(n=4[6 \%])$; vascular diseases $(\mathrm{n}=14[22 \%])$; cardiac valve diseases, including endocarditis $(n=4[6 \%])$; pulmonary diseases $(n=6$ $[10 \%])$; septicemia $(\mathrm{n}=3[5 \%])$; cancer $(\mathrm{n}=9[15 \%])$, gastrointestinal diseases $(n=4[6 \%])$; external causes/accidents $(\mathrm{n}=3[5 \%])$; and other unrelated causes of death $(\mathrm{n}=$ $2[3 \%])$. Survival for patients discharged alive is compared with an age-, sex-, and race-matched contemporary general population in Figure E1.

Early- and late-phase multivariate predictors for all-cause death and cardiovascular death are shown in Table 4. Note that the independent predictors of mortality in the early hazard phase included the same variables identified for hospital mortality by using logistic methods, plus "salvage surgery" and "crossclamp time" for all-cause death or "involvement of the carotid arteries" for cardiovascular death. The effect of age on early and long-term survival is represented in Figure $2, A$. Figure 2, $B$, shows the multivariable-adjusted 1-, 5-, $10-$, and 15 -year survival by year of surgical intervention.

\section{Freedom From Aortic Reoperation}

The mean aortic reoperation follow-up for patients discharged alive was $6.7 \pm 5.8$ years and was $91 \%$ complete (16 patients lost to follow-up). Freedom from aortic reoperation was $97 \% \pm 1 \%, 93 \% \pm 2 \%, 84 \% \pm 4 \%, 66 \% \pm$ $8 \%$, and $58 \% \pm 10 \%$ at $1,5,10,15$, and 20 years, respectively (Figure E2). Aortic reoperations included aortic valve replacements $(\mathrm{n}=2[8.3 \%])$, composite root replacements 
TABLE 2. Independent predictors of hospital death

\begin{tabular}{lcc}
\hline & Odds ratio $(\mathbf{9 5} \% \mathbf{C I})$ & $\boldsymbol{P}$ value \\
\hline Age & $2.40 \dagger(1.44-3.99)$ & $<.001$ \\
Serum creatinine & $4.67(1.18-18.52)$ & .03 \\
$\quad$ (in mg/dL) level & & \\
$\quad$ at admission* & & .003 \\
Syncope & $4.66(1.68-12.88)$ & .002 \\
Year of operation & $0.54 \ddagger(0.36-0.80)$ & \\
CI, Confidence interval. *Natural logarithmic transformation of serum creatinine (in \\
milligrams per deciliter). $\dagger$ Effect for 10-year difference in age. $\ddagger$ Effect for 5-year dif- \\
ference in year of operation.
\end{tabular}

$(\mathrm{n}=6[25 \%])$, arch repair $(\mathrm{n}=2[8.3 \%])$, thoracoabdominal aneurysm repairs $(\mathrm{n}=8[33 \%])$, abdominal aneurysm repairs $(n=4[17 \%])$, and surgical fenestrations of the abdominal aorta $(n=2[8.3 \%])$. Independent risk factors for aortic reoperation included year of surgical intervention (hazard ratio for 5-year increment, 2.30; 95\% confidence interval, 1.36-3.90; $P=.002$ ) and Marfan syndrome (hazard ratio, 5.97; 95\% confidence interval, $2.22-16.0 ; P<.001)$.

\section{Trends in Management}

Several variables were correlated with later date of surgical intervention, including more sophisticated diagnostic imaging modalities, such as CT scanning (Pearson $r=0.56$ ) and preprocedural transesophageal echocardiographic analysis $(r=0.57)$, open distal anastomosis $(r=0.73)$, antegrade cerebral perfusion $(r=0.41)$, use of antifibrinolytic agents $(r=0.64)$, and use of biologic glue $(r=0.51$; all $P$ $<.001)$. The time trend in competing diagnostic imaging modality, circulatory arrest use and cerebral protection, and surgical techniques used is shown in Figure 3. Overall, aortic angiography was used in $97(50 \%)$ patients, CT was used in $112(57 \%)$ patients, preprocedural transesophageal echocardiography was used in $130(67 \%)$ patients, and magnetic resonance imaging was used in $4(2 \%)$ patients. There was a trend toward more preservation of the aortic valve and less aortic valve replacement $(P=.05)$, whereas the percentage of composite root replacement remained stable.

\section{DISCUSSION}

Dissection of the ascending aorta remains one of the most common emergency procedures in cardiac surgery. Despite advances in stent graft technology, the relation of the ascending aorta to the aortic valve, coronary ostia, and arch vessels has left open repair as the only treatment in all but a few cases in select centers. Repair of AAAD typically involves a palliation of a more extensive disease process. It is uncommon that the operation removes the entire area of involved vasculature, with the focused goal being only to halt extension, improve branch flow, prevent rupture, and improve aortic valve function. Since the early series of surgical repair for $\mathrm{AAAD},{ }^{2}$ these goals have remained unchanged, but changes have occurred with the incorporation of new
TABLE 3. Independent predictors of postoperative stroke

\begin{tabular}{lcc}
\hline & Odds ratio $(\mathbf{9 5} \% \mathbf{C I})$ & $\boldsymbol{P}$ value \\
\hline Altered mental status & $2.96(1.22-7.17)$ & .02 \\
Aortic rupture & $3.64(1.29-10.25)$ & .01 \\
$\quad$ into pericardium & & .04 \\
Circulatory arrest & 1 & - \\
$\quad$ None (reference) & $3.22(1.12-9.26)$ & .01 \\
$\quad$ Without cerebral & & \\
$\quad$ protection & $1.33(0.40-4.41)$ & .5 \\
$\quad$ With cerebral protection & &
\end{tabular}

CI, Confidence interval.

preoperative testing methods, mainly $\mathrm{CT}$ and echocardiographic analysis, and use of improved techniques primarily related to myocardial and cerebral protection strategies.

This study was undertaken to better characterize both the effect of the evolution in management of this disease and the long-term survival after surgical treatment. We report the long-term results of aortic repair for AAAD over a 26-year period in a single large-volume referral center.

\section{Long-Term Survival}

Few studies present greater than 15-year survival data after repair for AAAD. In the early to the late 1990s, Crawford and associates, ${ }^{19}$ the Stanford group, ${ }^{20}$ and Bachet and colleagues ${ }^{4}$ reported 10 - and 15-year survival ranging from $37 \%$ to $56 \%$ and $24 \%$ to $46 \%$, respectively. Likewise, we report 10-, 15-, and 20-year survival of $55 \%, 48 \%$, and $30 \%$, respectively. The risk of death is markedly reduced after the perioperative period, although the survival of patients discharged alive remains lower compared with that of an age-, sex-, and race-matched general population.

\section{Patient Factors Affecting Survival}

Age is an established risk factor for early and late mortality. ${ }^{11,12,20}$ Some studies have focused on the outcomes of surgical intervention for AAAD in patients older than 70 years. ${ }^{24,25}$ Elderly patients differ markedly from their younger counterparts in terms of comorbidities, such as longstanding hypertension and atherosclerosis, and are more likely to present in cardiogenic shock.

The extent of renal impairment measured based on serum creatinine levels is a strong predictor of early mortality, as also reported by others. ${ }^{3,18,20,26}$ Acute renal failure is associated with mesenteric ischemia, drug-resistant hypertension, and aortic branch vessel compromise. ${ }^{26}$ Some have suggested that delayed surgical intervention or medical treatment with percutaneous fenestration or stenting has acceptable early outcomes in the setting of severe renal or intestinal malperfusion. ${ }^{27,28}$ However, this strategy has to be weighed against the increased risk of rupture, which is difficult to quantify, and generally poor results reported for medical therapy. ${ }^{15,18}$ 


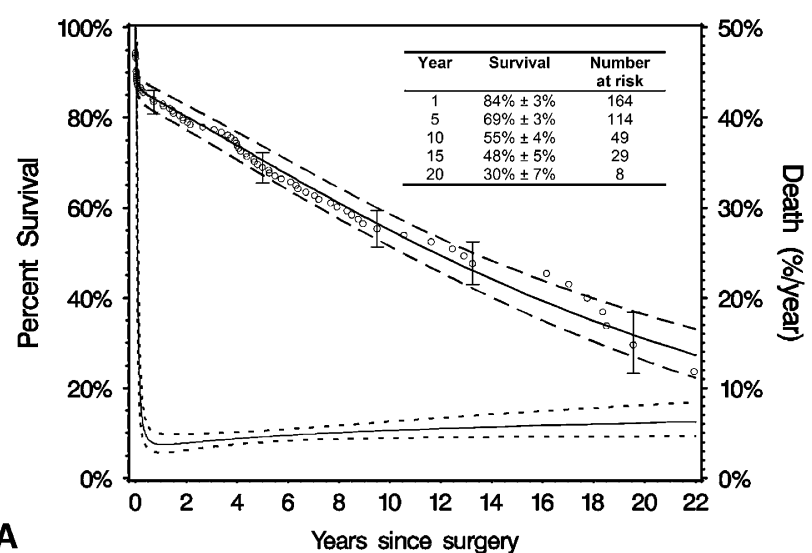

A

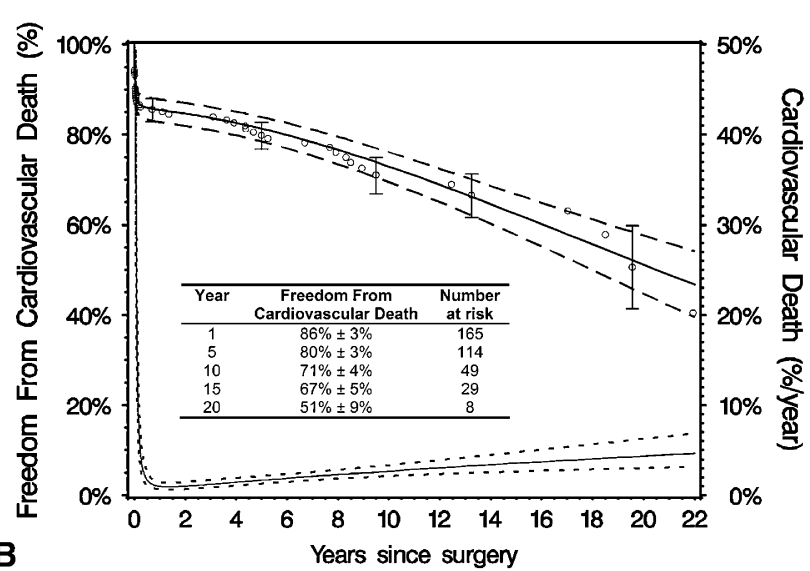

FIGURE 1. Survival (A) and freedom from cardiovascular death (B) after surgical intervention for all patients. Actuarial survival estimates with 1 standard deviation ( $68 \%$ confidence limits) and size of risk sets at 1,5 , 10,15 , and 20 years after surgical intervention are provided in the embedded table. The predicted survival from each parametric multiphase model is overlaid as a bold solid line in the same illustration, with a bold dashed line indicating 1 standard deviation. The thin solid line represents the hazard function (as percentage of death per year) for each model, with the dotted line indicating 1 standard deviation. The left vertical axis represents percentage survival, and the right vertical axis represents percentage death per year.

Late death was predicted based on peripheral vascular disease, which relates to the systemic involvement of arteries and prior vascular surgery. Patients with extensive peripheral vascular disease might be unable to compensate for reduced flow in the acute setting, leading to end-organ malperfusion, with a significant toll on mortality.

\section{Management Trends Associated With Improved Outcomes}

During this era, we observed incorporation of a variety of new methods, an associated reduction in hospital mortality from $21 \%$ in the early series to $4 \%$ recently, and improvement in adjusted 5-year survival from $65 \%$ to $89 \%$. We identified factors associated with this improvement, the most important of which were advances in diagnostic imag-
TABLE 4. Incremental risk factors for all-cause and cardiovascular death

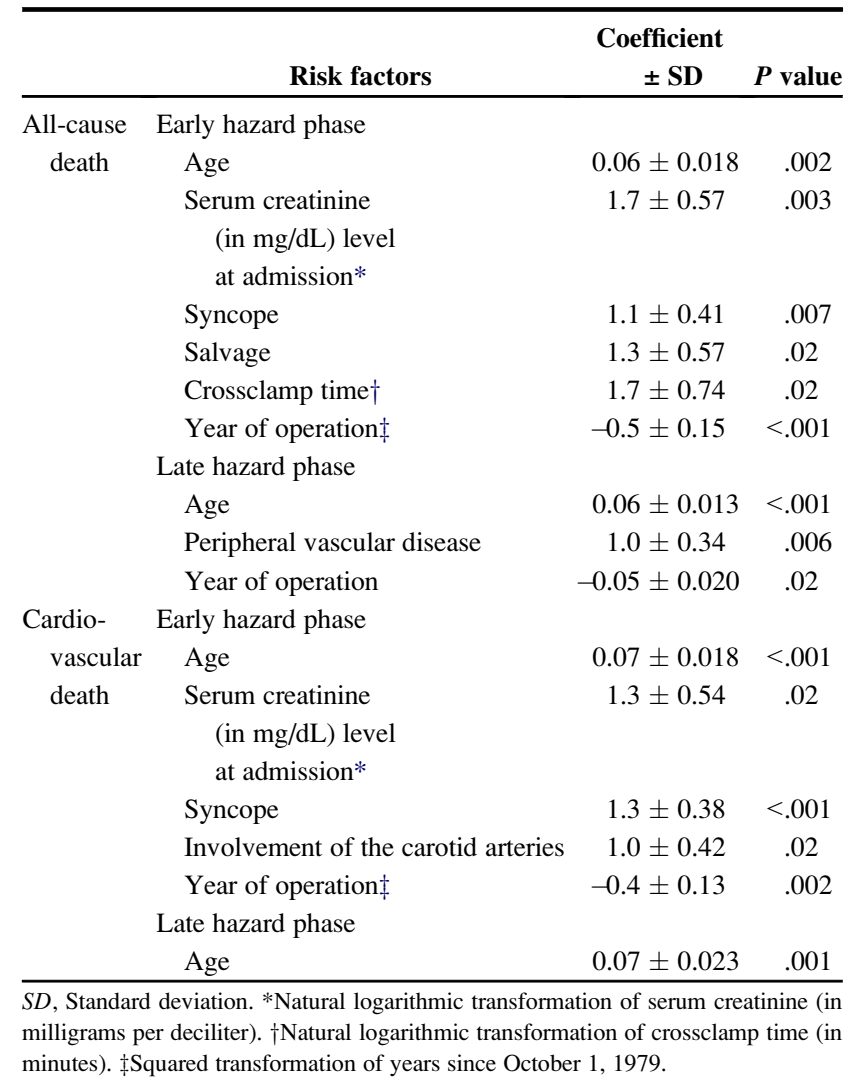

ing and improvement in ascending aortic repair techniques, including liberal use of hypothermic circulatory arrest with open distal anastomosis and cerebral protection techniques.

Diagnostic imaging modalities have undeniably improved over the last 2 decades, with a shift from invasive procedures with high contrast loads (aortography) to faster modalities with low contrast loads (CT) or applicable at bedside (echocardiography). As technology improved, aortography was rarely requested as a unique modality and was gradually combined with CT, preoperative echocardiography, or both. As we move forward in the years of treatment, angiography was seldom requested, whereas CT and echocardiography were generally combined. The International Registry of Acute Aortic Dissection ${ }^{29}$ and others ${ }^{30}$ have shown that all diagnostic modalities have a high sensitivity for ascending aortic dissection (CT, 93\%-100\%; transesophageal echocardiography, $90 \%-98 \%$; and aortography, $87 \%-88 \%$ ), but the false-negative rate is still considerable and mandates more than 1 modality to conclusively exclude the diagnosis. Magnetic resonance imaging was seldom used in our series.

Surgical techniques changed significantly during the study period. The practice of hypothermic circulatory arrest, visual inspection of the arch vessels, and open distal anastomosis was introduced in the 1980s and became routine soon afterward. In this series the initial arterial cannulation site of 

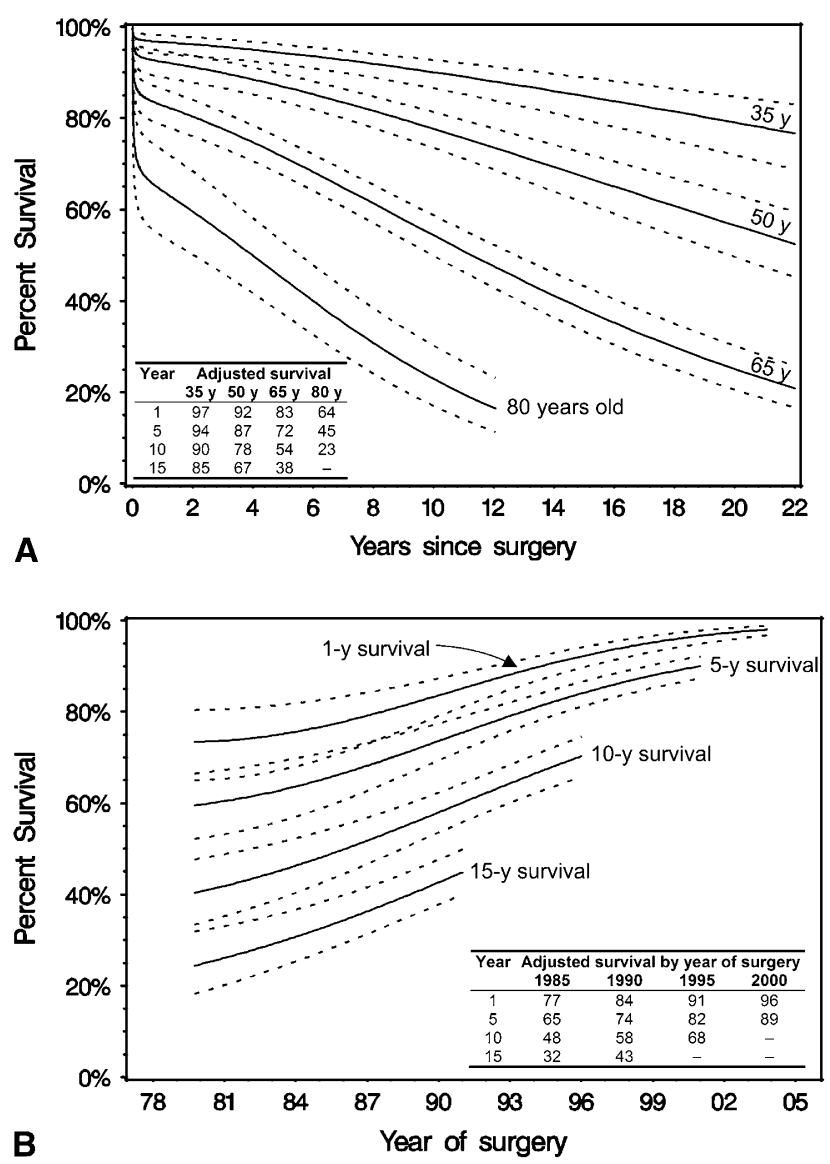

FIGURE 2. Multivariable-adjusted survival for patients aged 35, 50, 65, and 80 years (A) and multivariable-adjusted 1-, 5-, 10-, and 15-year survival by year of operation (B). Predictions are presented with 1 standard deviation (68\% confidence limits) and are truncated according to the maximum follow-up observed in each group. In these models continuous variables are fixed at their mean values (except for age in model A and year of operation for model B), and binary variables are set to zero.

choice was the femoral artery for rapid access and, more recently, conversion to antegrade perfusion through the aortic graft after distal anastomosis. Cerebral protection techniques were introduced in the 1990s, and antegrade cerebral protection is now more popular. David and coworkers ${ }^{5}$ suggested that avoidance of aortic crossclamping during cooling and resection of the primary tear improved early and late outcomes for AAAD. They further suggested a decrease in the incidence of stroke with this technique, which was not shown in this series. Instead, prior cerebral deficit, aortic rupture into the pericardium, and use of circulatory arrest without cerebral protection were shown to be independent predictors of stroke. In patients undergoing circulatory arrest and presenting without prior cerebral deficit or aortic rupture, the use of simple cerebral protection techniques was associated with a trend toward a decrease in the rate of stroke $(21 \%$ to $11 \%, P=.2)$. As antegrade perfusion techniques
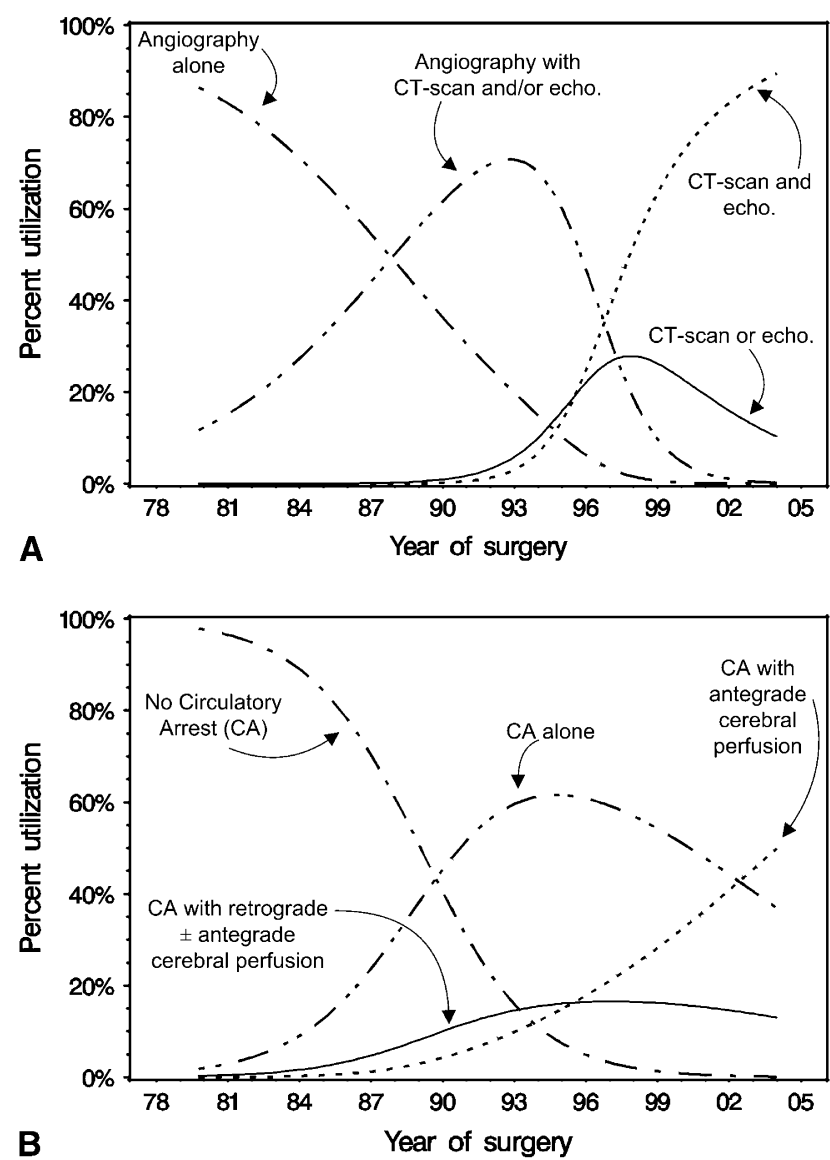

FIGURE 3. Changing patterns in management of acute ascending aortic dissection. The depictions highlight changes observed during the study period, with each curve representing the relative occurrence of mutually exclusive management strategies over time, adding up to $100 \%$ at every time point. Panel A demonstrates the change in use of preoperative diagnostic imaging modalities. Angiography is replaced gradually with the incorporation of computed tomography $(C T)$ and echocardiography (echo.). Panel B depicts the increasing use of circulatory arrest and antegrade/retrograde cerebral perfusion.

have evolved, we tend to use more frequently the axillary artery or the aortic arch and its branches as the initial cannulation site when the patient is hemodynamically stable.

Year of surgical intervention was also associated with improved late survival in addition to the effect seen on early survival. This might be related to better postoperative follow-up of these complicated patients with extensive vascular disease at specialized clinics or attentive follow-up from others. Diagnostic imaging techniques are becoming less invasive and more precise, both of which facilitate repeated follow-up examinations. The earlier detection of patients with vascular complications during follow-up and aggressive treatment of patients with Marfan syndrome might explain why patients are more likely to undergo an aortic reoperation in the later part of the series. The overall 
risk of aortic reoperation was low and comparable with that described in the published literature. ${ }^{4,7,8,12,20}$

\section{Limitations}

This study represents the experience of a large academic referral center over a 26-year period with a mean follow-up of $7.0 \pm 5.9$ years, or $8.1 \pm 5.6$ years in patients discharged alive, and might not be generalizable to others centers. As a referral center, many patients are transferred some hours after the initial presentation, and there might be some selection bias in the subpopulation of patients who actually underwent surgical intervention, knowing that there must have been a few who died or were deemed inoperable at some point in their evaluation.

Aortic reoperation follow-up was limited. Also, information regarding long-term outcome would ideally have included additional information on the patient's functional status collected prospectively.

Attempts to understand the factors that lead to observed changes over time were made difficult because of the fact that multiple "new" variables were appearing during the interval. These were highly correlated, and thus identifying (or excluding) any individual factor was made statistically impossible in some cases. Other factors might account for the improved early and late survival, including earlier referral, improved intensive care unit performance, and secondary or tertiary prevention measures.

\section{CONCLUSION AND CLINICAL INFERENCES}

Over the past 25 years, early and late outcomes for AAAD have improved in association with the incorporation of new methods and technologies, including echocardiography, CT imaging, use of open distal anastomosis, and improved cerebral protection techniques. The reported current early mortality of less that $5 \%$ was achieved by using a strategy that emphasizes avoidance of coronary angiography, combined use of CT and transesophageal echocardiographic data, a meticulous surgical technique involving routine use of hypothermic circulatory arrest with open distal anastomosis, and a conservative approach to aortic arch repair.

We are grateful for the research assistance provided by Sandra Debronkart, Karen E. Lynch, Jerene Bitondo, and Suyog Mokashi.

\section{References}

1. Gallo A, Davies RR, Coe MP, Elefteriades JA, Coady MA. Indications, timing, and prognosis of operative repair of aortic dissections. Semin Thorac Cardiovasc Surg. 2005; 17:224-35.

2. Debakey ME, Henly WS, Cooley DA, Morris GC Jr, Crawford ES, Beall AC Jr. Surgical management of dissecting aneurysms of the aorta. J Thorac Cardiovasc Surg. 1965;49:130-49.

3. Apaydin AZ, Buket S, Posacioglu H, Islamoglu F, Calkavur T, Yagdi T, et al. Perioperative risk factors for mortality in patients with acute type A aortic dissection. Ann Thorac Surg. 2002;74:2034-9.
4. Bachet J, Goudot B, Dreyfus GD, Brodaty D, Dubois C, Delentdecker P, et al. Surgery for acute type A aortic dissection: the Hopital Foch experience (19771998). Ann Thorac Surg. 1999;67:2006-9.

5. David TE, Armstrong S, Ivanov J, Barnard S. Surgery for acute type A aortic dissection. Ann Thorac Surg. 1999;67:1999-2001.

6. Ehrlich MP, Grabenwoger M, Kilo J, Kocher AA, Grubhofer G, Lassnig AM, et al. Surgical treatment of acute type A dissection: is rupture a risk factor? Ann Thorac Surg. 2002;73:1843-8.

7. Halstead JC, Meier M, Etz C, Spielvogel D, Bodian C, Wurm M, et al. The fate of the distal aorta after repair of acute type A aortic dissection. J Thorac Cardiovasc Surg. 2007;133:127-35

8. Kazui T, Washiyama N, Bashar AH, Terada H, Suzuki T, Ohkura K, et al. Surgical outcome of acute type A aortic dissection: analysis of risk factors. Ann Thorac Surg. 2002;74:75-81.

9. Lai DT, Miller DC, Mitchell RS, Oyer PE, Moore KA, Robbins RC, et al. Acute type A aortic dissection complicated by aortic regurgitation: composite valve graft versus separate valve graft versus conservative valve repair. J Thorac Cardiovasc Surg. 2003;126:1978-86.

10. Mehta RH, Suzuki T, Hagan PG, Bossone E, Gilon D, Llovet A, et al. Predicting death in patients with acute type a aortic dissection. Circulation. 2002;105:200-6.

11. Rampoldi V, Trimarchi S, Eagle KA, Nienaber CA, Oh JK, Bossone E, et al. Simple risk models to predict surgical mortality in acute type A aortic dissection: the International Registry of Acute Aortic Dissection score. Ann Thorac Surg. 2007;83:55-61.

12. Sabik JF, Lytle BW, Blackstone EH, McCarthy PM, Loop FD, Cosgrove DM. Long-term effectiveness of operations for ascending aortic dissections. $J$ Thorac Cardiovasc Surg. 2000;119:946-62.

13. Trimarchi S, Nienaber CA, Rampoldi V, Myrmel T, Suzuki T, Mehta RH, et al. Contemporary results of surgery in acute type A aortic dissection: the International Registry of Acute Aortic Dissection experience. J Thorac Cardiovasc Surg. 2005;129:112-22.

14. Westaby S, Saito S, Katsumata T. Acute type A dissection: conservative methods provide consistently low mortality. Ann Thorac Surg. 2002;73:707-13.

15. Tsai TT, Evangelista A, Nienaber CA, Trimarchi S, Sechtem U, Fattori R, et al. Long-term survival in patients presenting with type A acute aortic dissection: insights from the International Registry of Acute Aortic Dissection (IRAD). Circulation. 2006;114(suppl):I350-6.

16. Kallenbach K, Oelze T, Salcher R, Hagl C, Karck M, Leyh RG, et al. Evolving strategies for treatment of acute aortic dissection type A. Circulation. 2004; 110(suppl 1):II243-9.

17. Bavaria JE, Brinster DR, Gorman RC, Woo YJ, Gleason T, Pochettino A. Advances in the treatment of acute type A dissection: an integrated approach. Ann Thorac Surg. 2002;74(suppl):S1848-52.

18. Hagan PG, Nienaber CA, Isselbacher EM, Bruckman D, Karavite DJ, Russman PL, et al. The International Registry of Acute Aortic Dissection (IRAD): new insights into an old disease. JAMA. 2000;283:897-903.

19. Crawford ES, Kirklin JW, Naftel DC, Svensson LG, Coselli JS, Safi HJ. Surgery for acute dissection of ascending aorta. Should the arch be included? J Thorac Cardiovasc Surg. 1992;104:46-59.

20. Fann JI, Smith JA, Miller DC, Mitchell RS, Moore KA, Grunkemeier G, et al. Surgical management of aortic dissection during a 30-year period. Circulation. 1995; 92(suppl):II113-21.

21. Hilgenberg AD, Akins CW, Logan DL, Vlahakes GJ, Buckley MJ, Madsen JC, et al. Composite aortic root replacement with direct coronary artery implantation. Ann Thorac Surg. 1996;62:1090-5.

22. De Paepe A, Devereux RB, Dietz HC, Hennekam RC, Pyeritz RE. Revised diagnostic criteria for the Marfan syndrome. Am J Med Genet. 1996;62:417-26.

23. National Center for Health Statistics. U.S. decennial life tables for 1989-91. In: State life tables no. 22, Massachusetts., vol. II. Hyattsville, Md: National Center for Health Statistics; 1998.

24. Caus T, Frapier JM, Giorgi R, Aymard T, Riberi A, Albat B, et al. Clinical outcome after repair of acute type A dissection in patients over 70 years-old. Eur J Cardiothorac Surg. 2002;22:211-7.

25. Mehta RH, O'Gara PT, Bossone E, Nienaber CA, Myrmel T, Cooper JV, et al. Acute type A aortic dissection in the elderly: clinical characteristics, management, and outcomes in the current era. J Am Coll Cardiol. 2002;40:685-92.

26. Beckman JA, Mehta RH, Isselbacher EM, Bossone E, Cooper JV, Smith DE, et al. Branch vessel complications are increased in aortic dissection patients with renal insufficiency. Vasc Med. 2004;9:267-70.

27. Scholl FG, Coady MA, Davies R, Rizzo JA, Hammond GL, Kopf GS, et al. Interval or permanent nonoperative management of acute type A aortic dissection. Arch Surg. 1999;134:402-5. 
28. Slonim SM, Miller DC, Mitchell RS, Semba CP, Razavi MK, Dake MD. Percutaneous balloon fenestration and stenting for life-threatening ischemic complications in patients with acute aortic dissection. J Thorac Cardiovasc Surg. 1999; 117:1118-26.

29. Moore AG, Eagle KA, Bruckman D, Moon BS, Malouf JF, Fattori R, et al. Choice of computed tomography, transesophageal echocardiography, magnetic reso- nance imaging, and aortography in acute aortic dissection: International Registry of Acute Aortic Dissection (IRAD). Am J Cardiol. 2002;89:1235-8.

30. Shiga T, Wajima Z, Apfel CC, Inoue T, Ohe Y. Diagnostic accuracy of transesophageal echocardiography, helical computed tomography, and magnetic resonance imaging for suspected thoracic aortic dissection: systematic review and meta-analysis. Arch Intern Med. 2006;166:1350-6. 


\section{Appendix E1. Variables examined for association with outcomes \\ Patient variables}

Demography: age, sex

Presentation: interval between symptom onset and surgical intervention, hemodynamic state at the time of the operation (stable, unstable, or cardiogenic shock), characteristic of pain (sudden) and location (anterior, posterior, neck, abdominal, or extremities), altered mental status, focal neurologic deficit, paraplegia, syncope

Status of aorta: known extent of dissection (ascending or descending coronary arteries or carotid arteries), aortic valve regurgitation, bicuspid valve disease, aortic rupture into pericardium

Comorbidities: hypertension, coronary artery disease, previous myocardial infarction, electrocardiographically determined ST-segment elevation or depression, dyslipidemia, chronic heart failure, chronic obstructive pulmonary disease, smoking, peripheral vascular disease, Marfan disease, previous cardiac surgery, previous vascular surgery, serum creatinine level

\section{Procedure}

Cardiopulmonary bypass: cardiopulmonary bypass and crossclamp time, use of circulatory arrest and its duration

Operation: type of procedure performed (ascending aorta replacement [AAR] without valve procedure, AAR with either composite root replacement or aortic valve replacement, or AAR with aortic valve resuspension), hemiarch or total arch repair, open distal anastomosis, use of biologic glue, use of antifibrinolytic agent

Year of operation: years since October 1979

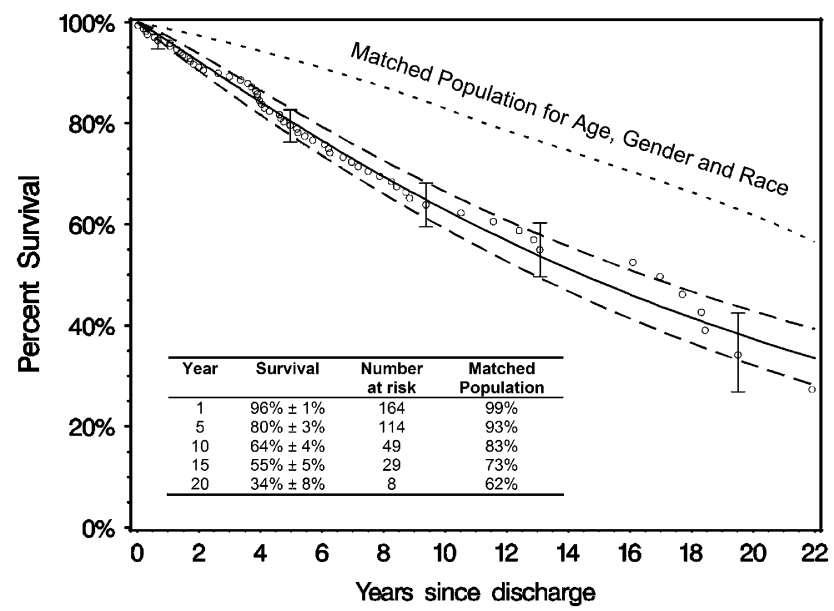

FIGURE E1. Survival after surgical intervention for all hospital survivors compared with Massachusetts' general population matched for age, sex, and race. Actuarial survival estimates with 1 standard deviation (68\% confidence limits) and size of risk sets at 1,5,10,15, and 20 years after surgical intervention are shown in the embedded table. The predicted survival from the parametric multiphase model is overlaid as a bold solid line in the same illustration, with a bold dashed line indicating 1 standard deviation. The bold dotted line represents the matched survival for the Massachusetts' general population.

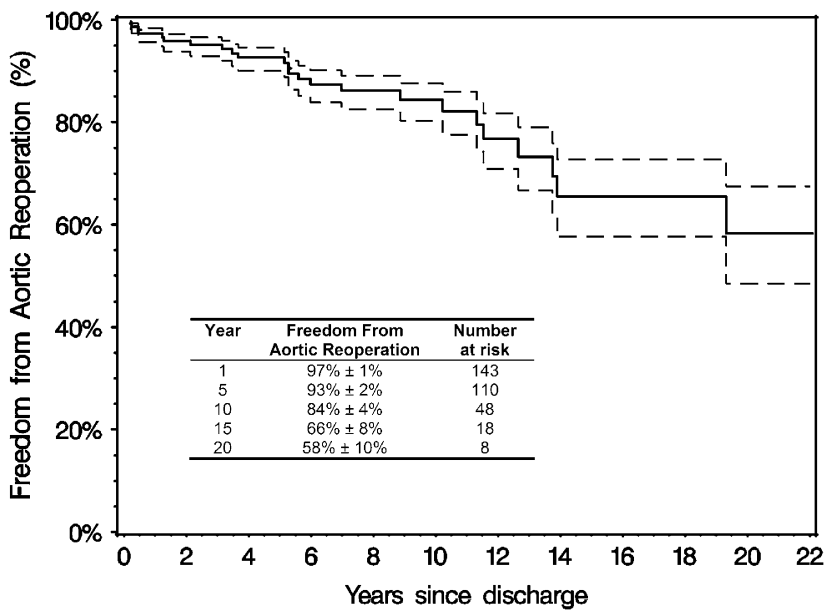

FIGURE E2. Freedom from aortic reoperation on the aorta or aortic valve for all hospital survivors. Actuarial survival estimates with 1 standard deviation (68\% confidence limits) and size of risk sets at 1, 5, 10, 15, and 20 years after surgical intervention are shown in the embedded table. 